\title{
Does the systemic administration of L- arginine affect dental implant stability in nicotine consumer dogs?
}

\author{
Bijan Movahedian', Mansour Rismanchian², Hooman Navaei ${ }^{3}$, Saeid Tavanafar $^{4 *}$ (D) and Soheil Koushaei ${ }^{5}$
}

\begin{abstract}
Background: Nicotine can have detrimental effects on dental implant osseointegration. This study aimed to evaluate the influence of systemic L-arginine supplement on the osseointegration of dental implants in nicotine consumer dogs.

Methods: Twelve 1-year Labrador Retriever dogs had their right and left third and fourth mandibular premolars removed, and the sockets were left to heal for 6 months. Dogs were randomly divided into three groups $(n=16)$ : group $1-0.2 \mathrm{mg} / \mathrm{kg}$ nicotine was injected twice daily; group $2-0.2 \mathrm{mg} / \mathrm{kg}$ nicotine was injected twice daily in addition to $200 \mathrm{mg} / \mathrm{kg}$ L-arginine capsules taken orally; and group 3-placebo. Forty-eight dental implants were inserted into the healed sockets of the dog's mandible and were assessed by implant stability quotient (ISQ) using resonance frequency analysis (RFA) during 4 weeks and insertion and removal torque value analysis.
\end{abstract}

Results: No implant failure occurred during the study period. The change in torque value between insertion and removal was similar in the placebo and nicotine+arginine consumer dogs $(p=0.276)$, which shows a positive effect of arginine supplementation in nicotine consumers. There was a significant difference in torque value change between nicotine+arginine vs. nicotine consumers $(p=0.049)$ and placebo vs. nicotine $(p=0.003)$. After 4 weeks, the placebo had the most significant improvement in torque value $(47.0 \pm 16.9)$, followed by nicotine+arginine (25.1 \pm 37.8$)$, and the worst torque value was for the nicotine group $(-5.7 \pm 24.0)$ pound per inch. The results show that except in the first week, there are significant differences in ISQ between the groups in different periods. ISQ in all of the groups has reduced at first but then increased over time. At the time of implant placement, insertion torque was significantly higher in the nicotine consumer group than the nicotine+arginine consumer group and placebo group $(p=0.020)$.

Conclusion: Arginine supplementation promotes bone healing and implant primary stability by improving dental implant osseointegration biomechanical characteristics.

Keywords: Implant stability quotient (ISQ), L-arginine, Nicotine, Osseointegration, Resonance frequency assessment (RFA)

\footnotetext{
* Correspondence: s.tavanafar@gmail.com; tavanafar@sums.ac.ir

${ }^{4}$ Department of Oral and Maxillofacial Surgery, School of Dentistry, Shiraz

University of Medical Sciences, Shiraz, Iran

Full list of author information is available at the end of the article
}

\section{Springer Open}

(c) The Author(s). 2021 Open Access This article is licensed under a Creative Commons Attribution 4.0 International License, which permits use, sharing, adaptation, distribution and reproduction in any medium or format, as long as you give appropriate credit to the original author(s) and the source, provide a link to the Creative Commons licence, and indicate if changes were made. The images or other third party material in this article are included in the article's Creative Commons licence, unless indicated otherwise in a credit line to the material. If material is not included in the article's Creative Commons licence and your intended use is not permitted by statutory regulation or exceeds the permitted use, you will need to obtain permission directly from the copyright holder. To view a copy of this licence, visit http://creativecommons.org/licenses/by/4.0/. 


\section{Background}

One of the critical factors in dental implant treatment success rate is mechanical stability after surgical insertion and functional masticatory loading [1-6]. The surrounding bone tissue after preparation of the implant site sustains severe inevitable damage. The injury to the surrounding bony tissue triggers active bone remodeling to form new bone neighboring the dental implants during the healing period [4].

One of the significant bone formation indicators and remodeling is the osteoblastic activity, required in dental implant osteointegration and healing of fractured bone. Previous reports show that several amino acids play a beneficial role in bone formation. Arginine, a semiessential amino acid, affects polyamine synthesis, stimulates growth hormone production, and improves endothelial function by enhancing nitric oxide formation [7-10].

Several risk factors, such as low quality and inadequate quantity of peri-implant bone, or various medical conditions, such as uncontrolled diabetes or osteoporosis, may endanger dental implant osteointegration and survival [11]. Another notable risk factor is tobacco smoking, attributed to dental implant failure [12]. Many authors studied tobacco smoking's significance in periimplant tissues and eventual implant failure [13-16]. The deleterious effect of tobacco smoking is mostly attributed to its nicotine content, although more than 4000 potentially toxic substances have been identified [17]. Nicotine is known to cause vasoconstriction and inhibit cellular healing responses [18]. Therefore, nicotine may harm dental implant osseointegration [19].

In the present study, we aimed to evaluate the effects of nicotine alone and in combination with arginine supplementation on early implant osseointegration.

\section{Methods}

\section{Sample size estimation}

We used previous trials, sixteen samples in each group required to reach a significant difference. Therefore, four dogs were designated in each group, and four implants for each dog have a 0.80 probability of having differences of $d=7.8$ when $\propto=0.05$.

\section{Ethical considerations for using animals}

All of the ethical issues concerning using the animals in a clinical trial by our university were followed. The ethical committee review board has confirmed the study protocol (IR.MUI.RESEARCH.REC.1398.005), and the reporting of the present study follows the ARRIVE checklist for all relevant items. All dogs were provided by the Isfahan University of Medical Sciences Animal Laboratory. We have used the minimum number of dogs required for this study, and all of the dogs survived after the study is finished.

\section{Experimental procedure}

Twelve 1-year-old male Labrador Retriever dogs were chosen for this study. Each dog, weighing approximately $30 \mathrm{~kg}$, had healthy dentition and periodontium. During the experiment, the animals were housed in private kennels with adequate ventilation and fed a soft diet. Each dog was anesthetized by giving $5 \mathrm{mg} / \mathrm{kg}$ IV ketamine hydrochloride, followed by halothane and nitrous oxide inhalation. After sterile prep and draping, the dog mouth was cleaned with $0.2 \%$ chlorhexidine for $30 \mathrm{~s}$. The throat pack using sterile gauze was placed around the endotracheal tube. Two percent lidocaine with 1:80,000 epinephrine was infiltrated on the buccal and lingual sides of the first and second mandibular premolars. Then, each tooth was sectioned and removed atraumatically (Fig. 1). Thus, a total number of four teeth in each dog were extracted for future implant placement. To ensure no remaining roots and to assess the teeth sockets, postoperative radiographs were obtained.

Six months after the tooth removal, the dog's mandible was examined clinically and radiographically, ready for implant placement (Fig. 2). Following the same anesthetic procedure, each dog was prepared for implant placement. After preparation and sterile draping of the

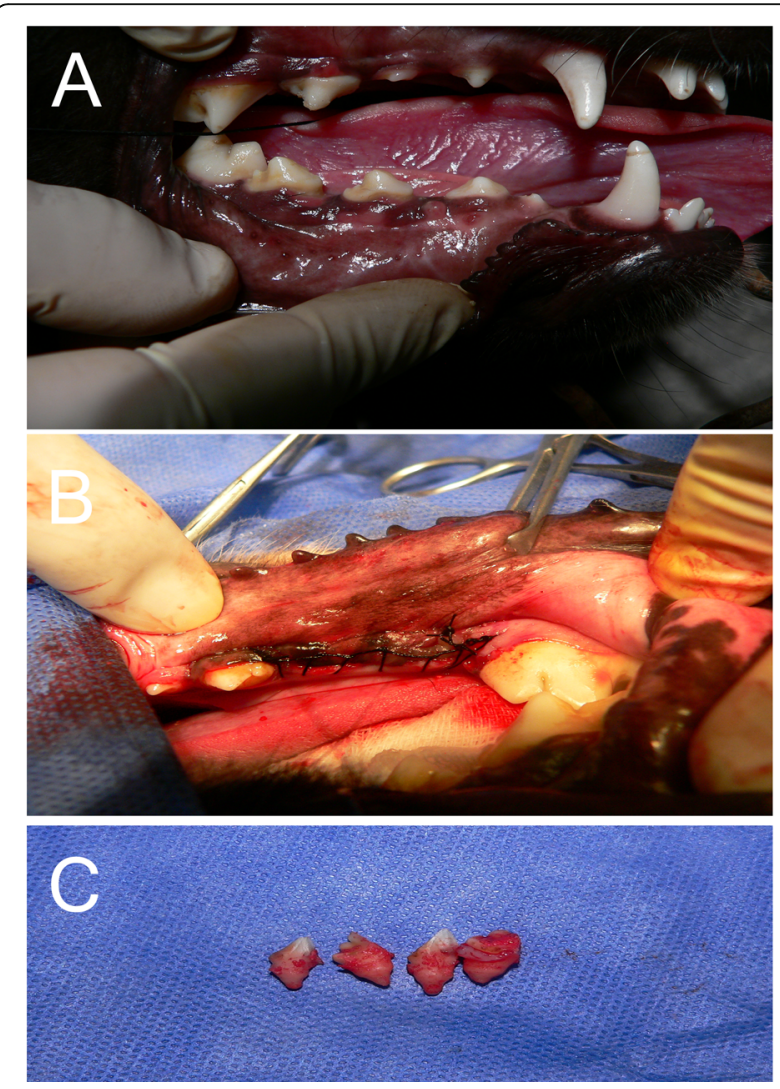

Fig. 1 a Preoperative mandibular premolars. b Surgically removed mandibular premolars and $\mathbf{c}$ removed dogs' teeth 
surgical field, surgical blade number 15 was used to make a crestal incision, and a mucoperiosteal flap was reflected (Fig. 2). Dental implants were inserted using the manufacturer's guidelines $(3.8 \mathrm{~mm} \times 10 \mathrm{~mm}$, Bio Horizons Implant System Inc., Birmingham, AL, USA, Fig. 2). During dental implant insertion, the required torque was measured and recorded in pound per inch using a Dial Measuring Torque Wrenches (Torqueleader, Gedore, Bramley, Guildford, UK) (Fig. 2). The insertion of dental implants continued until reaching the level of the crestal bone. The polished parts were not inserted into the bone. Smart peg of Ostell (Osstell, Integration Diagnostics Ltd., Gothenburg, Sweden) was inserted into the dental implants. Resonance frequency analysis (RFA) values were measured by the Osstell device (Fig. 2). This measures the implant stability quotient (ISQ) unit value shown as a scale of reading on the device screen. A periapical radiograph was taken to ensure the proper position of dental implants to the adjacent bone.

Dogs were placed on a soft diet for 1 week. Each group consisted of four dogs with four implants. Dogs were randomly assigned to three groups;
Nicotine-arginine group $(n=16)$ : dogs in this group received $2 \mathrm{mg} / \mathrm{kg} / 12 \mathrm{~h}$ nicotine subcutaneously and 200 $\mathrm{mg} / \mathrm{kg} /$ daily L-arginine by prefabricated capsules in their daily foods.

Nicotine group $(n=16)$ : dogs in this group received 2 $\mathrm{mg} / \mathrm{kg} / 12 \mathrm{~h}$ nicotine subcutaneously and prefabricated empty capsules.

Placebo $(n=16)$ : dogs in this group were receiving the normal saline while injecting nicotine to other dogs and prefabricated empty capsules in their daily foods.

ISQ was recorded at the time of implant insertion and every week for 4 weeks. Except for the first and last ISQ recordings, which were under general anesthesia, other recordings were under mild sedation of dogs (Fig. 2). During the 4-week experimental period, an experienced veterinary visited the dogs twice daily to ensure their health. After the fourth week, dogs were put under general anesthesia, and the fifth ISQ were recorded, and the reverse torque of the implant was measured using a manual torque wrench. Laboratory assistants recorded all of the measurements and were blinded to the group allocations of the study.
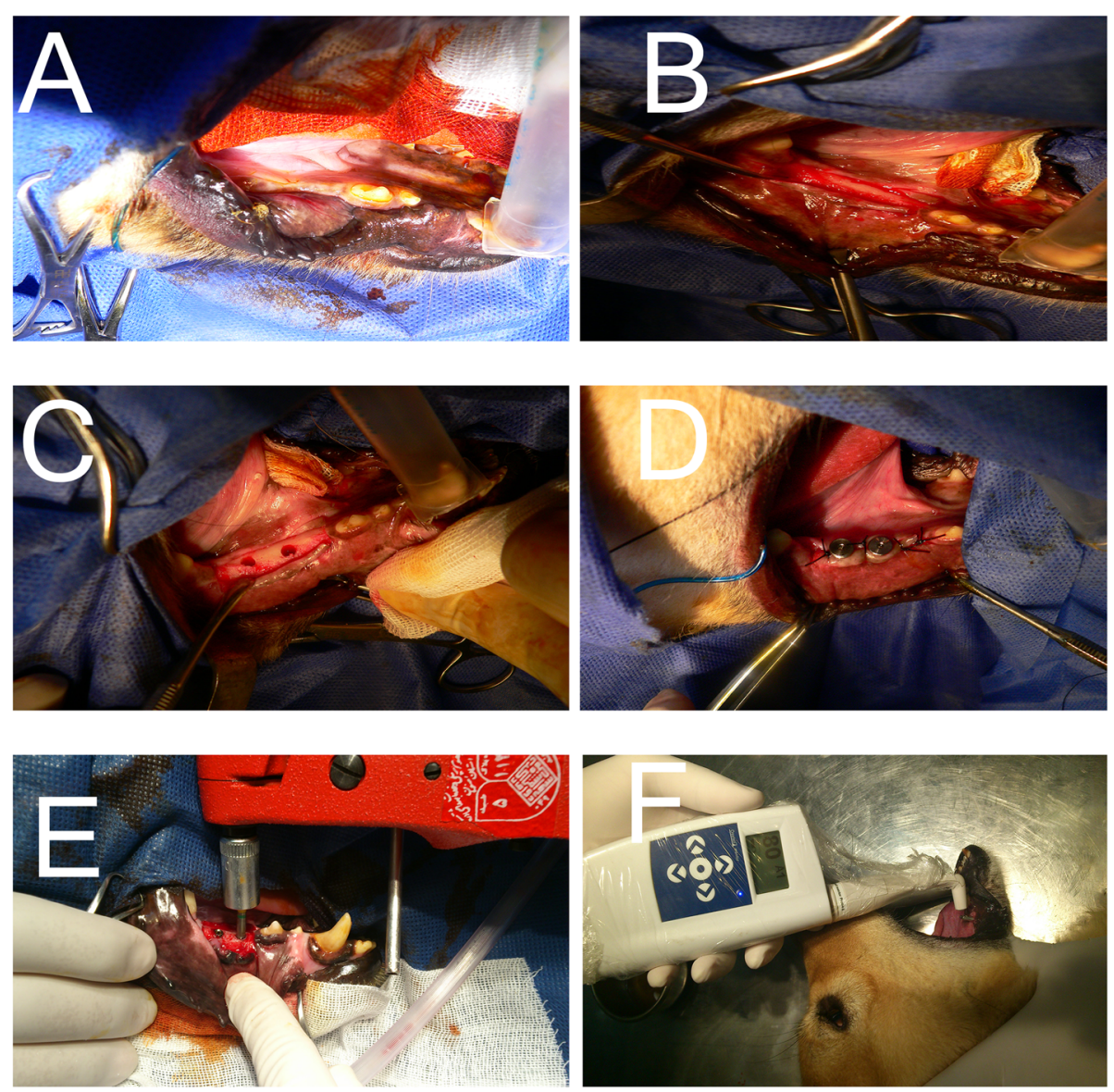

Fig. 2 Clinical view after 6 months of healing. Surgical exposure of the previous extraction site. Implant osteotomy preparation and implant placement. Torque value measurement and ISQ measurement 
Table 1 Comparison of torque between the 3 groups (pound per inch)

\begin{tabular}{|c|c|c|c|c|c|c|c|}
\hline \multirow[t]{2}{*}{ Torque } & \multicolumn{3}{|l|}{ Group } & \multicolumn{4}{|c|}{$p$ value } \\
\hline & a: nicotine & b: nicotine+L-arg & c: no nicotine no L-arg & Total & $a, b$ & $a, c$ & $\overline{b, c}$ \\
\hline Insertion & $85.6 \pm 17.5$ & $44.4 \pm 8.6$ & $60.5 \pm 9.1$ & 0.025 & 0.020 & 0.194 & 0.497 \\
\hline Removal & $79.87 \pm 27.6$ & $69.2 \pm 37.8$ & $107.5 \pm 16.5$ & 0.041 & 0.758 & 0.156 & 0.039 \\
\hline Change & $-5.7 \pm 24.0$ & $25.1 \pm 37.8$ & $47.0 \pm 16.9$ & 0.004 & 0.049 & 0.003 & 0.276 \\
\hline$p$ value & 0.521 & 0.103 & $<0.001$ & & & & \\
\hline
\end{tabular}

Data were presented as mean $\pm \mathrm{SD}$; column $p$ value, extract from one-way ANOVA and Tukey post hoc; row $p$ value, extract from paired $t$ test

\section{Statistical analysis}

Data were presented as mean \pm SD for continuous variables. In this analysis, one-way analysis of variance (ANOVA) with Tukey post hoc test, repeated measure (RE) ANOVA, and paired $t$ test with two sides at the $5 \%$ level of significance were used. All the analyses were performed using the Statistical Package for Social Sciences version 16.0 (SPSS Inc., Chicago, IL, USA).

\section{Results}

In this study, twelve dogs, each with four dental implants, were studied over 4 weeks. No implant failure occurred during the study. Torque value analysis is presented in Table 1. At the time of implant placement, insertion torque was significantly higher in the nicotine consumer group than the nicotine+arginine consumer group and placebo group $(p=0.020)$. After 4 weeks, dental implants' removal torque was significantly different between nicotine+arginine consumer dogs and placebo, and placebo had higher removal torque ( $p=$ 0.039). When comparing insertion torques and removal torques of all the three groups, only the placebo group was significantly different $(p<0.001)$. Nicotine consumer dogs had also gained less torque upon removal than the other two groups during the 4 weeks. The other two groups had increased torque during the study period. Although there were no significant differences between nicotine+arginine consumers and placebo, placebo had higher torque values in two periods of time, which shows adverse effects of nicotine on implant

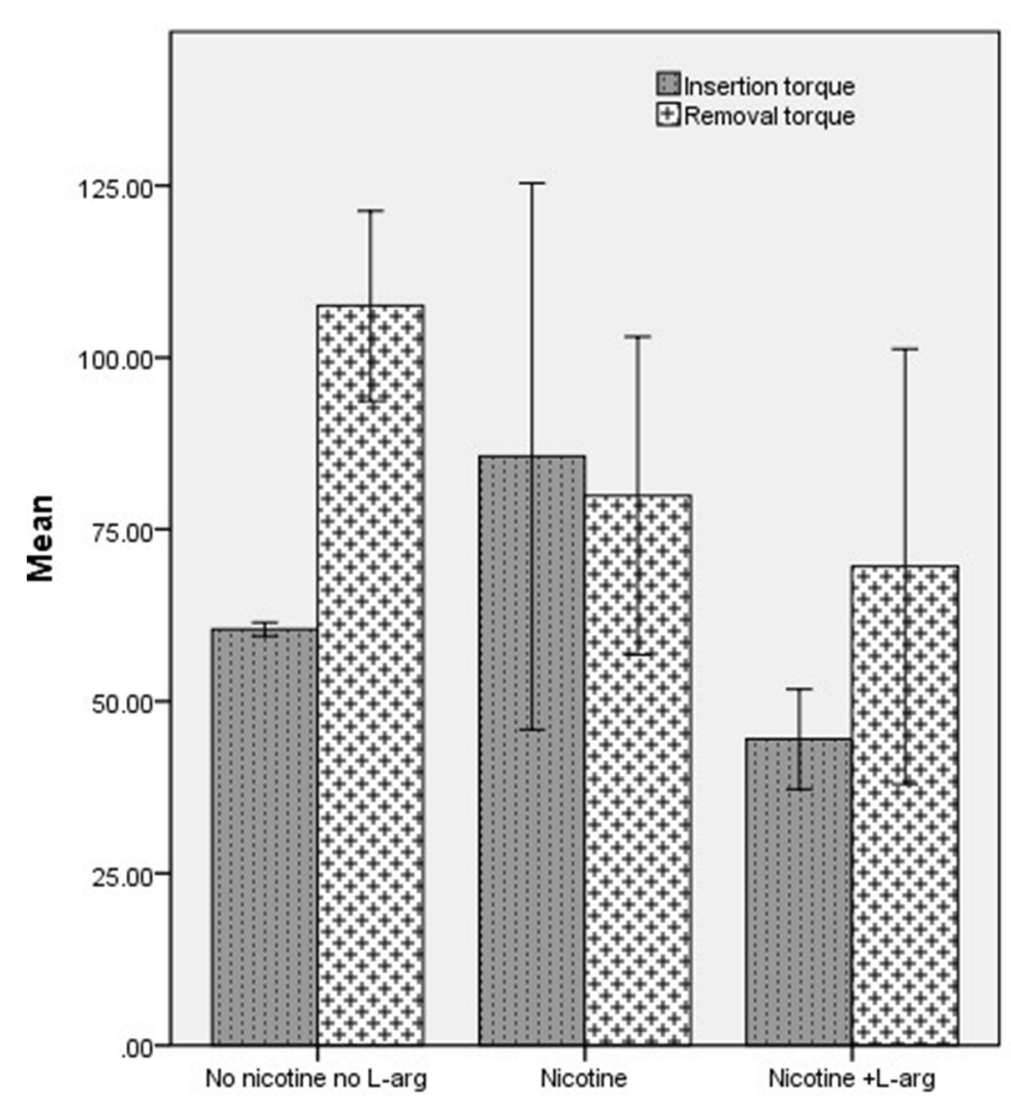

Fig. 3 The mean of torque at insertion and removal time in the three groups 
Table $\mathbf{2}$ Comparison of ISQ between the 3 groups in five different times

\begin{tabular}{|c|c|c|c|c|c|c|c|c|}
\hline \multirow[t]{2}{*}{ ISQ } & & \multicolumn{3}{|l|}{ Group } & \multicolumn{4}{|c|}{$p$ value } \\
\hline & & a: nicotine & b: nicotine $+\mathrm{L}$-arg & c: no nicotine no L-arg & Total & $a, b$ & $a, c$ & $b, c$ \\
\hline Baseline & & $78.0 \pm 2.6$ & $73.5 \pm 2.3$ & $72.2 \pm 3.1$ & 0.001 & 0.009 & 0.001 & 0.609 \\
\hline Week 1 & & $74.8 \pm 4.5$ & $67.4 \pm 8.3$ & $65.4 \pm 4.1$ & 0.035 & 0.077 & 0.048 & 0.970 \\
\hline Week 2 & & $68.0 \pm 7.3$ & $61.6 \pm 6.7$ & $68.5 \pm 4.17$ & 0.072 & - & - & - \\
\hline Week 3 & & $77.3 \pm 4.5$ & $63.5 \pm 4.9$ & $63.5 \pm 9.0$ & 0.001 & 0.003 & 0.003 & $>0.999$ \\
\hline Week 4 & & $80.0 \pm 3.7$ & $68.1 \pm 9.5$ & $75.6 \pm 6.9$ & 0.011 & 0.009 & 0.452 & 0.114 \\
\hline \multirow[t]{2}{*}{$p$ value } & Linear & 0.158 & 0.213 & 0.616 & & & & \\
\hline & Quadratic & $<0.001$ & 0.016 & $<0.001$ & & & & \\
\hline
\end{tabular}

Data were presented as mean \pm SD; column $p$ value, extract from one-way ANOVA and Tukey post hoc; row $p$ value, extract from repeated measure ANOVA

torque, and using arginine might have compensated for adverse effects of nicotine. However, still, it is not comparable to when no nicotine is used (Fig. 3).

ISQ values are presented in Table 2 . The results show that except in the first week, there are significant differences in ISQ between the groups in different periods. At the time of insertion, there were significant differences between the nicotine consumer group and nicotine+arginine consumer group $(p=0.009)$ and nicotine consumers group and placebo group $(p=0.001)$. In the first week, only nicotine consumers and placebo showed a significant difference $(p=0.048)$. In the third week, there was a significant difference between nicotine consumers compared to nicotine+arginine consumers $(p=$ 0.003 ) and nicotine consumers compared to placebo ( $p$ $=0.003)$, but in the fourth week, there were only significant differences between the nicotine consumer group and the nicotine+arginine consumers $(p=0.009)$. ISQ in all of the groups has reduced at first but then increased over time. This process can be seen in all of the three groups (Fig. 4).

\section{Discussion}

The present study tried to evaluate the effects of nicotine alone and in combination with arginine to find out any alterations in mechanical properties of early dental implant osseointegration in dogs' mandible. The present study showed that nicotine could negatively affect the mechanical properties of the dental implant. Arginine partially compensated for the negative effect of nicotine. Nicotine alone or in combination with arginine resulted

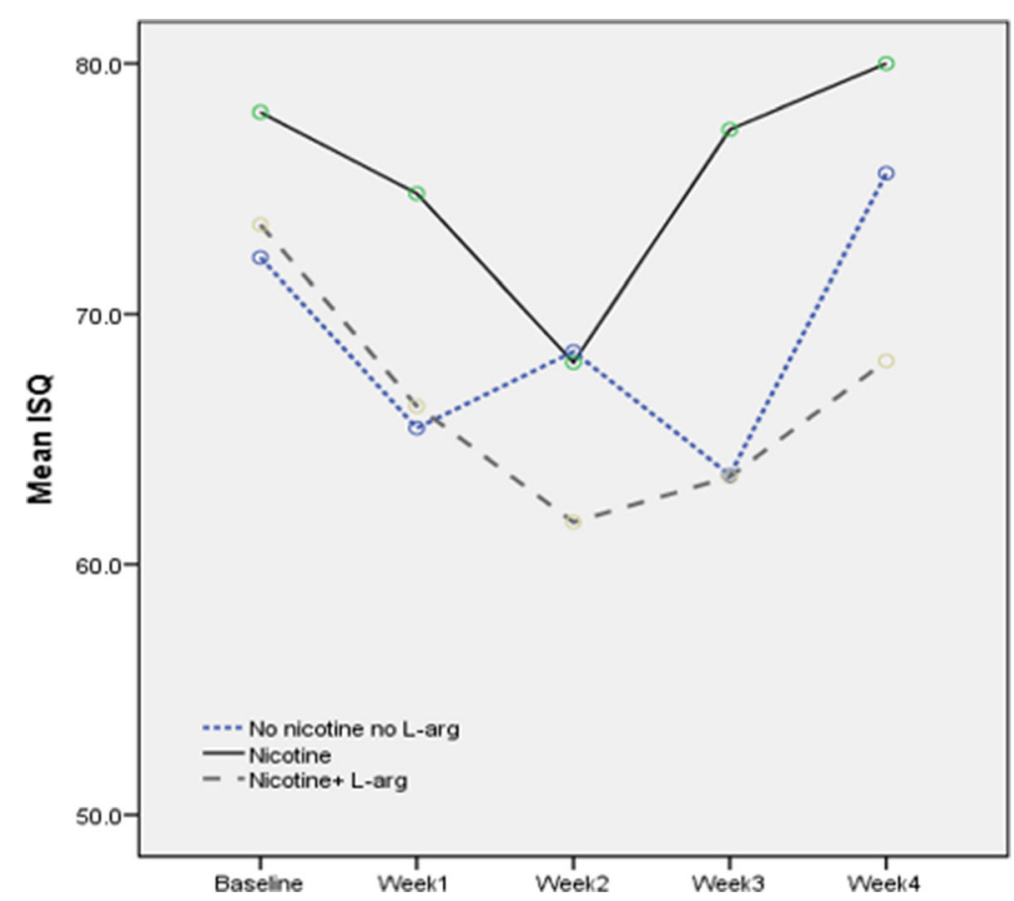

Fig. 4 The mean ISQ was measured every week for 4 weeks in the three study groups 
in inferior mechanical properties compared to placebo during 4 weeks of post-implantation.

Nicotine is known to reduce the proliferation of red blood cells, macrophages, and fibroblasts. It also affects tissue perfusion and healing by increasing platelet adhesion and vasoconstriction. Sympathomimetic action of nicotine stimulates epinephrine and norepinephrine release, which consequently causes vasoconstriction and limited tissue perfusion. Contemplating these effects, nicotine is expected to debilitate potential bone healing at the bone and implant interface. At 8 weeks, nicotine consumer rats showed that the bone matrix-related gene expression was downregulated, and bone formation around implants was decreased [20].

Berley et al. [21] found that bone-to-implant contact is decreased in rats receiving nicotine compared to control. Soares et al. showed a decrease in bone formation around hydroxyapatite implants placed in the tibia and femur of rats receiving nicotine compared to rats receiving water or alcohol [22]. On the other hand, Pereira et al. demonstrated that nicotine increases bone-forming enzymes' synthesis and enhances osteoblasts' growth and differentiation [23].

Cesar-Neto et al. found no difference in rats' bone healing receiving or not receiving nicotine [24]. Balatsoaka et al. demonstrated an increase in bone density from 2 to 4 weeks around implants in the rabbit receiving nicotine [25]. These contradictory results might be due to cell cultures used and other methodological differences between these studies.

In the present study, nicotine has resulted in reduced removal torque compared to the placebo. Arginine supplementation has increased torque over 4 weeks, and there was no significant difference in removal torque between the nicotine and arginine consumer groups compared to placebo.

The bone around dental implants continues an active remodeling even after 5 years of implantation. Studies that investigated bone healing around dental implants after few weeks to 20 years or more implant functioning show that masticatory loads stimulate continuous remodeling after primary healing of an implant $[6,26]$. The active remodeling is known to affect the bone quality, that is, the bone-implant contact area and the quality of the bone, which is influenced by the amount and distribution of collagen and minerals in the bone around dental implants. Thus, continuous remodeling contributes to implant mechanical stability over the functional years of a dental implant $[4,26,27]$. Although 4 weeks might be a short period in the present study, there was an increase in ISQ and removal torque values in all of the groups.

There is a strong correlation between the degree of tissue mineralization, as an indicator of bone quality, and mechanical properties of the bone [3, 28].
Incompletely mineralized newly formed bone due to bone remodeling adjacent to the implant is anticipated to be mechanically inferior to pre-existing bony tissues away from the implant. Therefore, In the present study, we have used mechanical measurements to evaluate the implant stabilities. Although histometric analysis would have given us more information, dental implants' biomechanical behavior is one of the most important prognostic factors and an essential indicator of osseointegration.

Collagen is the dominant constituent of bony tissues that control the viscoelastic properties $[3,29,30]$. Under the load, protein fibrils of collagen show time-dependent deformation.

During the bone mineralization process, minerals are gradually arranged along the collagen fibrils and eventually create the mineral-collagen complex's mechanical integrity. Thus, bone remodeling by changing the bone tissue composition alters bone's mechanical properties such as viscoelastic characteristics-further studies are required to investigate bone's viscoelastic properties around dental implants.

Many attempts have been made to find superior dental materials and growth agents to promote bone quality and quantity around and at the implant-bone interface. One of several agents is amino acids, which have been suggested that can hasten bone healing by enhancing local blood supply, stimulating growth factor, and increasing collagen synthesis [31].

In vitro studies have shown that amino acids such as arginine promote osteoblastic growth and differentiation by stimulating insulin growth factor-1 secretion [32-34]. Others found that arginine, lysine, and glycine are associated with collagen synthesis [10, 35]. Goel et al. [36] found that $2 \mathrm{~g} /$ day $\mathrm{L}$-arginine increased bone mineral density by $11.6 \%$ in osteoporotic women for 2 years.

In the present study, arginine was supplemented to nicotine consumer dogs to help in the osseointegration of dental implants. Arginine supplementation's beneficial effect might be due to arginine's potential therapeutic effect on bone healing by enhancing nitrous oxide production and collagen synthesis. Cell proliferation precedes the synthetic phase of osteogenic bone-derived osteoblasts [10].

\section{Conclusion}

Under the present study condition, arginine supplementation promotes bone healing and implant primary stability by improving dental implant osseointegration biomechanical characteristics. Further studies are required, paving the way for possible clinical application in the context of early and immediate implant loading.

Abbreviations

ISQ: Implant stability quotient; ANOVA: One-way analysis of variance 


\section{Acknowledgements}

None.

\section{Authors' contributions}

BM, MR, and SK: methodology. HN and ST: operations. ST, HN, and SK: writing - original draft preparation. BM and MR: writing - review and editing. BM, MR, and SK: supervision and project administration. The author(s) read and approved the final manuscript.

\section{Funding}

Not applicable.

\section{Availability of data and materials}

The datasets used and/or analyzed during the current study are available from the corresponding author on reasonable request.

\section{Ethics approval and consent to participate}

We obtained written informed consent to use the animals in our study from the animal institution (our university animal laboratory). Ethical behaviors with experimental animal model code: IR.MUI.RESEARCH.REC.1398.005 granted by Isfahan University of Medical Sciences, Isfahan, Iran.

\section{Consent for publication}

Not applicable.

\section{Competing interests}

The authors declare that they have no competing interests.

\section{Author details}

${ }^{1}$ Department of Oral and Maxillofacial Surgery, School of Dentistry, Isfahan University of Medical Sciences, Isfahan, Iran. ${ }^{2}$ Department of Prosthodontics, Dental Implants Research Center, School of Dentistry, Isfahan University of Medical Sciences, Isfahan, Iran. ${ }^{3}$ Department of Oral and Maxillofacial Surgery, School of Dentistry, Isfahan University of Medical Sciences, Esfahan, Iran. ${ }^{4}$ Department of Oral and Maxillofacial Surgery, School of Dentistry, Shiraz University of Medical Sciences, Shiraz, Iran. ${ }^{5}$ Department of Oral and Maxillofacial Surgery, School of Dentistry, Qazvin University of Medical Sciences, Qazvin, Iran.

Received: 19 December 2020 Accepted: 25 January 2021

Published online: 10 February 2021

\section{References}

1. Isidor F (2006) Influence of forces on peri-implant bone. Clin Oral Implants Res 17:8-18

2. Hoshaw SJ, Brunski JB, Cochran GVB (1994) Mechanical loading of Brånemark implants affects interfacial bone modeling and remodeling. Int J Oral Maxillofac Implant 9:1-33

3. Donnelly E, Boskey AL, Baker SP, van der Meulen MCH (2010) Effects of tissue age on bone tissue material composition and nanomechanical properties in the rat cortex. J Biomed Mater Res A 92:1048-1056

4. Kim D-G, Huja SS, Tee BC et al (2013) Bone ingrowth and initial stability of titanium and porous tantalum dental implants. Implant Dent 22:399-405

5. Brunski JB (1988) Biomaterials and biomechanics in dental implant design. Int J Oral Maxillofac Implants 3:85-97

6. Baldassarri M, Bonfante E, Suzuki M, et al (2012) Mechanical properties of human bone surrounding plateau root form implants retrieved after 0.3-24 years of function. 2015-2021.

7. Loche S, Carta D, Muntoni A et al (1993) Oral administration of arginine enhances the growth hormone response to growth hormone-releasing hormone in short children. Acta Paediatr 82:883-884

8. Ströhle A, von Bibra H, Hahn A (2016) L-arginine and vascular health. Med Monatsschr Pharm 39:515-520

9. Wu G, Bazer FW, Davis TA et al (2009) Arginine metabolism and nutrition in growth, health and disease. Amino Acids 37:153

10. Fini M, Torricelli $P$, Giavaresi $G$ et al (2001) Effect of L-lysine and L-arginine on primary osteoblast cultures from normal and osteopenic rats. BioMed Pharmacother 55:213-220

11. Neukam FW, Flemmig TF (2006) Local and systemic conditions potentially compromising osseointegration. Clin Oral Implants Res 17:160-162
12. Al Amri MD, Kellesarian SV, Abduljabbar TS et al (2017) Comparison of periimplant soft tissue parameters and crestal bone loss around immediately loaded and delayed loaded implants in smokers and non-smokers: 5-year follow-up results. J Periodontol 88:3-9

13. Turri A, Rossetti $P$, Canullo $L$ et al (2016) Prevalence of peri-implantitis in medically compromised patients and smokers: a systematic review. Int J Oral Maxillofac Implants 31:111-118

14. Moraschini V, Barboza E dS. P (2016) Success of dental implants in smokers and non-smokers: a systematic review and meta-analysis. Int J Oral Maxillofac Surg 45:205-215.

15. Manzano G, Montero J, Martín-Vallejo J et al (2016) Risk factors in early implant failure. Implant Dent 25:272-280

16. Chrcanovic BR, Albrektsson T, Wennerberg A (2015) Smoking and dental implants: a systematic review and meta-analysis. J Dent 43:487-498

17. Hoffmann DHI, Hoffmann I (1997) The changing cigarette, 1950-1995. J Toxicol Environ Health 50:307-364

18. Daftari TK, Whitesides TE, Heller JG et al (1994) Nicotine on the revascularization of bone graft. Spine (Phila Pa 1976) 19:904-911

19. Javed F, Rahman I (2000) Romanos GE (2019) Tobacco-product usage as a risk factor for dental implants. Periodontol 81:48-56

20. Yamano S, Berley JA, Kuo WP et al (2010) Effects of nicotine on gene expression and osseointegration in rats. Clin Oral Implants Res 21:1353-1359

21. Berley J, Yamano S, Sukotjo C (2010) The effect of systemic nicotine on osseointegration of titanium implants in the rat femur. J Oral Implantol 36:185-193

22. Soares EV, Fávaro WJ, Cagnon VHA et al (2010) Effects of alcohol and nicotine on the mechanical resistance of bone and bone neoformation around hydroxyapatite implants. J Bone Miner Metab 28:101-107

23. Pereira ML, Carvalho JC, Peres F et al (2008) Behaviour of human osteoblastic cells cultured on plasma-sprayed titanium implants in the presence of nicotine. Clin Oral Implants Res 19:582-589

24. César-Neto JB, Duarte PM, Sallum EA et al (2003) A comparative study on the effect of nicotine administration and cigarette smoke inhalation on bone healing around titanium implants. J Periodontol 74:1454-1459

25. Balatsouka D, Gotfredsen K, Lindh CH, Berglundh T (2005) The impact of nicotine on osseointegration: an experimental study in the femur and tibia of rabbits. Clin Oral Implants Res 16:389-395

26. Piattelli A, Artese L, Penitente E, laculli F, Degidi M, Mangano C, Shibli JA, Coelho PG, Perrotti V, lezzi G (2014) Osteocyte density in the peri-implant bone of implants retrieved after different time periods ( 4 weeks to 27 years). J Biomed Mater Res B Appl Biomater 102:239-43. https://doi.org/10.1002/jbm.b.33000.

27. Anchieta RB, Baldassarri M, Guastaldi F et al (2014) Mechanical property assessment of bone healing around a titanium-zirconium alloy dental implant. Clin Implant Dent Relat Res 16:913-919

28. Mulder L, Koolstra JH, den Toonder JMJ, van Eijden TMGJ (2007) Intratrabecular distribution of tissue stiffness and mineralization in developing trabecular bone. Bone 41:256-265

29. Kim DG, Kwon HJ, Jeong YH et al (2016) Mechanical properties of bone tissues surrounding dental implant systems with different treatments and healing periods. Clin Oral Investig 20:2211-2220

30. Morris MD, Mandair GS (2011) Raman assessment of bone quality. Clin Orthop Relat Res 469:2160-2169

31. Sinha S, Goel SC (2009) Effect of amino acids lysine and arginine on fracture healing in rabbits: a radiological and histomorphological analysis. Indian J Orthop 43:328-334

32. Yang J, Zhang $X$, Wang W, Liu J (2010) Insulin stimulates osteoblast proliferation and differentiation through ERK and PI3K in MG-63 cells. Cell Biochem Funct 28:334-341

33. Liu Z, Jeppesen PB, Gregersen S et al (2008) Dose- and glucose-dependent effects of amino acids on insulin secretion from isolated mouse islets and clonal INS-1E beta-cells. Rev Diabet Stud 5:232-244

34. Chevalley T, Rizzoli R, Manen D et al (1998) Arginine increases insulin-like growth factor-l production and collagen synthesis in osteoblast-like cells. Bone 23:103-109

35. Meléndez-Hevia E, De Paz-Lugo P, Cornish-Bowden A, Cárdenas ML (2009) A weak link in metabolism: the metabolic capacity for glycine biosynthesis does not satisfy the need for collagen synthesis. J Biosci 34:853-872

36. Goel SC, JhA G, Singh anant, et al (2014) Role of $L$ arginine in treatment of osteoporosis. Int J Orthop 1:177-180.

\section{Publisher's Note}

Springer Nature remains neutral with regard to jurisdictional claims in published maps and institutional affiliations. 